\title{
A ROTATING PLUG MODEL OF FRICTION STIR WELDING HEAT TRANSFER
}

\author{
J. K. Raghulapadu ${ }^{1}$, J. Peddieson ${ }^{1}$, G. R. Buchanan ${ }^{2}$ \\ Departments of ${ }^{1}$ Mechanical Engineering and ${ }^{2}$ Civil and Environmental Engineering \\ Tennessee Technological University \\ Cookeville, Tennessee 38505
}

\author{
A. C. Nunes \\ Materials Processes and Manufacturing Department \\ NASA Marshall Space Flight Center \\ Huntsville, Alabama 35812
}

\begin{abstract}
A simplified rotating plug model is employed to study the heat transfer phenomena associated with the friction stir welding process. An approximate analytical solution is obtained based on this idealized model and used both to demonstrate the qualitative influence of process parameters on predictions and to estimate temperatures produced in typical friction stir welding situations.
\end{abstract}

\footnotetext{
* Corresponding author, e-mail: jpeddieson(tntech.edu, tel: (931) 372-3274, fax: (931) 372-6340
} 


\section{Introduction}

The primary purpose of this paper is to develop an approximate closed form solution applicable to the description of the heat transfer phenomena associated with the friction stir welding (FSW) process. This solution extends classical quasi-steady conduction heat transfer solutions for moving sources and hot spots to the modeling of FSW heat transfer. A secondary purpose is to use this solution to discuss certain qualitative aspects of FSW heat transfer. It is not the purpose of this paper to present detailed process simulations, An interesting finding is that a well known elementary. conduction heat transfer solution can be employed to estimate the maximum temperatures occurring in FSW.

A schematic of a typical FSW setup is shown in Fig. 1. Plates A and B are to be welded along their common boundary (the weld seam). Plates A and B taken together will be referred to as the workpiece. The workpiece has a length $\mathrm{L}$ and a width $\mathrm{W}$. Typically the workpiece is placed on a backup plate (anvil) and clamped rigidly to prevent lateral movement. Figure 1 also introduces a cartesian coordinate system $(\mathrm{x}, \mathrm{y}, \mathrm{z})$ and a cylindrical polar coordinate system $(r, \ldots, z)$ which will both be used in later analysis.

The weld is performed by a pintool. It traverses the weld seam with a speed $\mathrm{V}$ (starting at $i$ and ending at $f$ ) while rotating with an angular velocity $\Omega$. Figure 2 shows a schematic of a typical pintool, which consists of a rotating shaft and a rotating nib as depicted. The bottom surface of the shaft, which is in contact with the workpiece, is called the shoulder. The workpiece has a thickness $H$. The nib extends a depth D $(\mathrm{D} \leq \mathrm{H})$ into the workpiece. If $\mathrm{D}=\mathrm{H}$ the configuration is called "full penetration" while if

$\mathrm{D}<\mathrm{H}$ the configuration is called "partial penetration". The shoulder radius is $\mathrm{R}_{s}$ and the 
nib radius is $R\left(R<R_{s}\right)$. To begin the process, the nib is brought into contact with the weld seam in the vicinity of $i$ (see Fig.1). The contact between the nib and the top surface of the workpiece results in the frictional generation of heat. This softens the nearby metal and allows the nib to be forced into the workpiece to achieve the configuration depicted in Fig. 2 with full shoulder contact. A region of yielded (but not melted) metal forms in the immediate vicinity of the pintool which reaches a maximum temperature around $80 \%$ to $90 \%$ of the melting temperature of the workpiece. The rotation of the pintool plastically "stirs" metal from each plate into the other. As the pintool moves down the seam the metal cools leaving a completed weld. In the vicinity of $f$ (see Fig. 1), the nib runs off the plate (usually onto a runoff tab), is withdrawn leaving a hole, or, in the case of the retractable pintool invented at Marshall Space Flight Center, is retracted into the shaft without leaving a hole at the end of the seam.

Some representative papers dealing with thermal and mechanical modeling of FSW are those by Chao and Qi [1], Ulysse [2], Hyoe et al. [3], Chao et al. [4], Colegrove and Shercliff [5], Song and Kovacevic [6,7], Schmidt et al. [8], Chen and Kovacevic [9], Soundararajan et al. [10], Schmidt and Hattel [11], Colegrove and Shercliff [12], Cho et al. [13], and Zhang et al. [14]. Several other pertinent publications are referred to therein. An inspection of the above cited references shows that there are two basic approaches to FSW heat transfer simulations. A considerable portion of the heating which maintains a FSW process is provided by mechanical dissipation heating in the yielded region. Thus, the most accurate approach is to perform a combined thermomechanical (FSW-TM) simulation (see, for instance, [2]; [5], and [9-14]). This involves numerical methodologies that require extremely fine grids in the pintool vicinity in order to capture the details of 
the small yielded region and, thus, are computationally intensive. Because of this, many investigators (see, for instance, [1], [3], [4], and [6-8]) have performed thermal simulations (FSW-T) in which the heating is regarded as prescribed. Since the heating depends on the details of the mechanical behavior in the yielded region, FSW-T models are not as complete as FSW-TM models. Nevertheless, they are desirable for certain purposes (preliminary parametric studies, for example) because of their reduced computational requirements. The present contribution presents an analytical FSW-T model which is complementary to the numerical FSW-T models reported in the above cited references. It employs a simplified "rotating plug" model of FSW to develop an approximate closed form solution describing FSW heat transfer. This solution is used to evaluate qualitatively the influence of process parameters on predictions.

\section{Governing Equations}

The details of a typical actual FSW setup are shown in Figs. 1 and 2. The rotating plug FSW heat transfer model is created by introducing several idealizations. These are enumerated below.

First, the pintool is idealized as a nib with $\mathrm{D}=\mathrm{H}$ (rotating plug). The frictional heating associated with the rotating plug surface is adjusted to be representative of the frictional heating associated with all surfaces of the actual pintool (shoulder bottom, nib side, and nib bottom). The procedure used to accomplish this is discussed in the "Numerical Examples": section below.

Second, the process is treated as steady from the viewpoint of the pintool. The justification for this is as follows. Typically, the inequalities $R_{s}<<W$ and $R_{s}<<L$ both hold. Thus, except in the immediate vicinities of $i$ and $f$, the changes of distance between 
the pintool and the ends of the weld seam are of secondary importance. Also, classical solutions for heat transfer associated with moving hot spots and sources (see, for instance, Carslaw and Jaeger [15]) indicate that, after a brief transient period, a steady state is established from the viewpoint of the hotspot or source. The same physics is assumed to apply in the present situation. Thus the process is viewed from the pintool as one in which the pintool rotates with angular velocity U relative to the workpiece which moves past it with speed $\mathrm{V}$ and the coordinate systems shown in Fig. 1 are regarded as having their origins at the intersection of the fixed pintool centerline and the bottom of the workpiece.

Third, because of the inequalities stated in the previous paragraph, it is believed that the exact geometry of the workpiece outer boundary in the $x, y$ plane will have a minor effect on heat transfer in the vicinity of the pintool. Therefore, in order to facilitate closed form solutions, the workpiece is idealized as being infinite in the $\mathrm{x}, \mathrm{y}$ plane.

Fourth, the mechanical behavior exhibited by the process is highly idealized. In reality the workpiece will exhibit a yielded region close to the pintool and an unyielded region away from the pintool. Mechanical dissipation in the yielded region produces much of the heating responsible for FSW. In the present work, the yielded region is idealized as being coincident with the rotating plug surface and the heating due to mechanical dissipation is represented by a heat flux at the rotating plug surface. In addition the workpiece motion is idealized as a uniform stream parallel to the weld seam, thus neglecting the motion in the yielded region necessary for the metal to flow around the plug. 
The simplifications discussed above produce the rotating plug FSW heat transfer model to be employed herein. For this model, the standard heat conduction equation (see, for instance, [15]) becomes

$$
\partial_{\mathrm{r}}^{2} \mathrm{~T}+\partial_{\mathrm{r}} \mathrm{T} / \mathrm{r}+\partial_{\mathrm{e}}^{2} \mathrm{~T} / \mathrm{r}^{2}+\partial_{\mathrm{z}}^{2} \mathrm{~T}=-\mathrm{V}\left(\cos (\theta) \partial_{\mathrm{r}} \mathrm{T}-\sin (\theta) \partial_{\theta} \mathrm{T} / \mathrm{r}\right) / \alpha
$$

when written in cylindrical polar coordinates. The left hand side of Eq. (1) represents heat conduction while the right hand side represents heat convection by a uniform stream. In Eq. (1), $\mathrm{T}$ is the absolute temperature and a is the workpiece thermal diffusivity. The corresponding radial, transverse, and axial heat flux components can be written

$\mathrm{q}_{\mathrm{r}}=-\mathrm{k} \partial_{\mathrm{T}} \mathrm{T}, \quad \mathrm{q}_{\mathrm{e}}=-\mathrm{k} \partial_{\dot{\mathrm{e}}} \mathrm{T} / \mathrm{r}, \quad \mathrm{q}_{\mathrm{z}}=-\mathrm{k} \partial_{\mathrm{z}} \mathrm{T}$

where $\mathrm{k}$ is the workpiece thermal conductivity.

To obtain the heat flux which represents the interaction between the pintool and the workpiece, it is assumed that the entire pintool surface is acted on by a transverse shear stress at the yield value $\hat{o}_{0}$. In the terminology of Schmidt et al. [8], this is a "stick" model as opposed to a "slide" model. Experimental data are presented in [8] suggesting indirectly that the "stick" model is superior.

The mechanical power generated by the moment associated with this yield stress acting on an area element $\mathrm{dA}$ of the rotating plug surface is

$$
\mathrm{dp}=\hat{\mathrm{o}}_{0} \mathrm{R} \Omega \mathrm{dA}
$$

which can be converted into an equivalent pintool surface radial heat flux

$$
\mathrm{q}_{\mathrm{r}}(\mathrm{R}, \theta, \mathrm{z})=\mathrm{dp} / \mathrm{dA}=\hat{\mathrm{o}}_{0} \mathrm{R} \Omega
$$


Combining Eq. (2a) (here and subsequently "a" denoted the first of a set of equations, "b" the second, etc.) evaluated at $\mathrm{r}=\mathrm{R}$ with Eq. (4) produces the expression

$$
\partial_{\mathrm{r}} \mathrm{T}(\mathrm{R}, \theta, \mathrm{z})=-\hat{\mathrm{o}}_{0} \mathrm{R} \Omega / \mathrm{k}
$$

The yield stress is a function of both temperature and strain rate. In the present model the pintool angular velocity can be used to characterize the strain rate so it could equally well be stated that the yield stress depends on both temperature and angular velocity. Since the angular velocity is constant during a FSW operation, the dependence on temperature will be focused on herein. Typical maximum FSW temperatures are $80 \%$ to $90 \%$ of the melting temperature $T_{M}$, as mentioned earlier. In order to facilitate closed form solutions, it was decided to use the straight line idealization

$$
\hat{\mathrm{o}}_{0}=\hat{\mathrm{o}}_{0,0}\left(1-\mathrm{T} / \mathrm{T}_{\mathrm{M}}\right)
$$

In the high temperature FSW range. Equation (6) captures both the temperature softening effect and the vanishing of the yield stress at the melting temperature in the context of a linear model. Results presented by Colegrove and Shercliff [12] suggest that the inclusion of temperature softening or some equivalent mechanism is necessary to avoid unrealistically high temperature predictions. The quantity $\hat{o}_{0,0}$ is a parameter having the dimensions of stress which would be chosen to make Eq. (6) provide the best representation of a given set of yield stress/temperature data in the FSW range at a given strain rate. Combining Eqs. (5) and (6) yields

$$
\partial_{\mathrm{r}} \mathrm{T}(\mathrm{R}, \theta, \mathrm{z})=-\hat{\mathrm{o}}_{0,0} \mathrm{R} \Omega\left(1-\mathrm{T}(\mathrm{R}, \theta, \mathrm{z}) / \mathrm{T}_{\mathrm{M}}\right) / \mathrm{k}
$$


Because heating is also provided by sliding friction over some portion of the shoulder surface (not included explicitly in the present model), some additional adjustment of the parameter $\hat{o}_{0,0}$ will normally be needed in order to obtain realistic predictions. The heating model employed herein has many features in common with those discussed in [68].

It should be mentioned that the closed form solution to be obtained subsequently could be readily imbedded in an iteration scheme which would deal with an arbitrary yield stress/temperature relation by treating both $\hat{o}_{0,0}$ and $T_{M}$ appearing in Eq. (7) as adjustable parameters and recomputing them at each iteration to get the best straight line fit to the yield stress/temperature behavior in the vicinity of the temperature calculated at the previous iteration. This subject is not pursued further herein.

The workpiece/anvil and workpiece/top environment interactions will be represented berein by the respective heat transfer coefficients $h_{0}$ and $h_{H}$. Then the definition of a heat transfer coefficient, combined with the evaluation of the appropriate element of Eq. (2) at the appropriate surface leads to

$\partial_{z} T(r, \theta, 0)=h_{0}\left(T(r, \theta, 0)-T_{A}\right) / k$

$\partial_{z} T(r, \theta, H)=h_{H}\left(T_{\infty}-T(r, \theta, H)\right) / k$

where $T_{\infty}$ is the environmental temperature and $T_{A}$ is the anvil temperature. Also

$\mathrm{T}(\mathrm{r}, \theta, \infty)=\mathrm{T}_{\infty}$

Equations (1) and (7) - (10) form the boundary value problem

$$
\partial_{\mathrm{r}}^{2} \mathrm{~T}+\partial_{\mathrm{r}} \mathrm{T} / \mathrm{r}+\partial_{\mathrm{e}}^{2} \mathrm{~T} / \mathrm{r}^{2}+\partial_{z}^{2} \mathrm{~T}+\mathrm{P}\left(\cos (\theta) \partial_{\mathrm{r}} \mathrm{T}-\sin (\theta) \partial_{\theta} \mathrm{T} / \mathrm{r}\right) / \mathrm{R}=0
$$




$$
\begin{aligned}
& \partial_{\mathrm{r}} \mathrm{T}(\mathrm{R}, \theta, \mathrm{z})-\mathrm{T}(\mathrm{R}, \theta, \mathrm{z}) /(\varepsilon \mathrm{R})=-\mathrm{T}_{\mathrm{M}} /(\varepsilon \mathrm{R}) \\
& \mathrm{T}(\mathrm{r}, \theta, \infty)=\mathrm{T}_{\infty} \\
& \partial_{z} \mathrm{~T}(\mathrm{r}, \theta, 0)-\mathrm{B}_{0} \mathrm{~T}(\mathrm{r}, \theta, 0) / \mathrm{H}=-\mathrm{B}_{0} \mathrm{~T}_{\mathrm{A}} / \mathrm{H} \\
& \partial_{z} \mathrm{~T}(\mathrm{r}, \theta, \mathrm{H})+\mathrm{B}_{\mathrm{H}} \mathrm{T}(\mathrm{r}, \theta, \mathrm{H}) / \mathrm{H}=\mathrm{B}_{\mathrm{H}} \mathrm{T}_{\infty} / \mathrm{H}
\end{aligned}
$$

In Eqqs. (11)-(15)

$$
\mathrm{P}=\mathrm{VR} / \alpha, \quad \mathrm{B}_{\mathrm{H}}=\mathrm{h}_{\mathrm{H}} \mathrm{H} / \mathrm{k}, \quad \mathrm{B}_{\mathrm{o}}=\mathrm{h}_{\mathrm{o}} \mathrm{H} / \mathrm{k}, \quad \varepsilon=\mathrm{kT}_{\mathrm{M}} /\left(\mathrm{fo}_{\mathrm{o}, \mathrm{o}} \Omega \mathrm{R}^{2}\right)
$$

are the respective Peclet number, Biot numbers associated with the top and bottom workpiece surfaces, and a quantity having no accepted name which will be called the "mechanical dissipation number" herein. A correction factor $f$ has been inserted into the mechanical dissipation number to account for the fact that the surface area of the rotating plug is less than the surface area of the actual pintool.

It should be noted that the Peclet number is a dimensionless measure of the welding translation speed, the Biot numbers are dimensionless measures of the degree of thermal interaction between the workpiece and its surroundings, and the mechanical dissipation number is a composite dimensionless quantity involving the welding rotation speed and the yielding behavior of the workpiece. Thus, the effect of process parameters on predictions can be characterized efficiently in terms of these dimensionless numbers.

\section{Approximate Closed Form Solution for Small Peclet and Biot Numbers}

Inspection of the various parametric combinations discussed in the previously cited literature reveals Peclet and Biot numbers in the respective ranges $0.1-0.3$ and 0.001-0.02. This information suggests that approximations based on the smallness of the 
Peclet and Biot numbers should be useful. Further, the range of Biot numbers suggests equating the Biot numbers to zero in Eqs. (14) and (15). This creates boundary conditions describing insulated top and bottom workpiece surfaces and allows a solution of the form $T=T(r$, è $)$ which reduces Eqs. (11), (12), and (13) to the respective forms

$$
\begin{aligned}
& \partial_{\mathrm{r}}^{2} \mathrm{~T}+\partial_{\mathrm{r}} \mathrm{T} / \mathrm{r}+\partial_{\dot{\mathrm{e}}}^{2} \mathrm{~T} / \mathrm{r}^{2}=-\mathrm{P}\left(\cos (\grave{\mathrm{e}}) \partial_{\mathrm{r}} \mathrm{T}-\sin (\grave{\mathrm{e}}) \partial_{\mathrm{e}} \mathrm{T} / \mathrm{r}\right) / \mathrm{R} \\
& \partial_{r} T(R, \grave{e})-T(R, \grave{e}) /(a ̊ R)=-T_{M} /(a ̊ R), T(\infty, \grave{e})=T_{\infty}
\end{aligned}
$$

Equations (17) describe a two dimensional version of the FSW-T problem. Examples of other recent two dimensional FSW simulations are provided by the papers of Cho et al. [13] and Zhang et al. [14].

A simple approximate closed form solution of the problem defined by Eqs. can be found for small Peclet numbers using an approach similar to that discussed by Trivedi and Srinivasan [16]. Omitting the details for the sake of brevity and using the small argument form of a Bessel function, the result can be written in the reduced temperature form

$$
\left(\mathrm{T}-\mathrm{T}_{\infty}\right) /\left(\mathrm{T}_{\mathrm{M}}-\mathrm{T}_{\infty}\right)=\psi(\mathrm{r}) \exp (-\operatorname{Pr} \cos (\theta) /(2 \mathrm{R}))
$$

where

$\left.\psi=\mathrm{K}_{\mathrm{o}}(\mathrm{Pr} / 2 \mathrm{R})\right) /(\varepsilon+\ln (2 / \mathrm{P}))$

In Eq. (19) $\mathrm{K}_{0}$ is a zeroth order modified Bessel function of the second kind. Near the pintool the small argument form of_can be used to further simplify Eq. (18) to

$$
\left(\mathrm{T}-\mathrm{T}_{\infty}\right) /\left(\mathrm{T}_{\mathrm{M}}-\mathrm{T}_{\infty}\right)=\psi_{0}(\mathrm{r}) \exp (-\operatorname{Pr} \cos (\theta) /(2 \mathrm{R}))
$$

where 
$\psi_{0}=\ln (2 \mathrm{R} /(\mathrm{Pr})) /(\varepsilon+\ln (2 / \mathrm{P}))$

The combination of Eqs. (20) and (21) with the exponential term omitted is just the reduced temperature associated with axisymmetric steady heat conduction in a cylindrical shell having respective inner and outer radii

$r_{i}=R, \quad r_{o}=2 / P$

and respective inner and outer temperatures

$\mathrm{T}_{\mathrm{i}}=\mathrm{T}_{\infty}+\left(\mathrm{T}_{\mathrm{M}}-\mathrm{T}_{\infty}\right) \ln (2 / \mathrm{P}) /(\varepsilon+\ln (2 / \mathrm{P})), \quad \mathrm{T}_{0}=\mathrm{T}_{\infty}$

(see, for instance, Incropera and DeWitt [17]). Thus, this elementary solution can be adapted to the description of the temperature distribution near the pintool in FSW.

Evaluating Eq. (21) at the pintool surface yields

$\phi=1 /(1+\xi)$

where

$\phi=\psi_{0}(\mathrm{R}), \quad \xi=\varepsilon / \ln (2 / \mathrm{P})$

It can be shown that, to the order of approximation inherent in Eq. (18), $\phi$ represents the average reduced temperature on the pintool surface. For $0.1<\mathrm{P}<0.3$ the maximum and minimum reduced temperatures will differ from the average by a maximum of about $15 \%$. The elementary cylindrical shell solution can, therefore be used to estimate the maximum temperatures produced in a FSW operation.

The combination of Eqs. (18) and (19) can be rewritten as

$$
\left(\mathrm{T}-\mathrm{T}_{\infty}\right) /\left(\mathrm{T}_{\mathrm{M}}-\mathrm{T}_{\infty}\right)=\mathrm{QK}_{\mathrm{o}}(\operatorname{Pr} /(2 \mathrm{R})) \exp (-\operatorname{Pr} \cos (\theta) /(2 \mathrm{R})) /(2 \pi \mathrm{k})
$$


This is identical to the classical solution for quasi-steady conduction due to a moving line source of heat (see, for instance, Carslaw and Jaeger [15]) with source strength (power per unit length)

$\mathrm{Q}=2 \pi \mathrm{k}\left(\mathrm{T}_{\mathrm{M}}-\mathrm{T}_{\infty}\right) /(\varepsilon+\ln (2 / \mathrm{P}))$

Thus, except in the immediate vicinity of the pintool, the workpiece cannot distinguish between mechanical dissipation and line sources of heat.

The combination of Eqs. (18) and (19) can also be written

$$
\left(\mathrm{T}-\mathrm{T}_{\infty}\right) /\left(\mathrm{T}_{\mathrm{M}}-\mathrm{T}_{\infty}\right)=\mathrm{K}_{0}\left(\mathrm{P}\left(\mathrm{x}^{2}+\mathrm{y}^{2}\right)^{1 / 2} /(2 \mathrm{R})\right) \exp (-\mathrm{Px} /(2 \mathrm{R})) /(\varepsilon+\ln (27 \mathrm{P}))
$$

For large distances from the pintool, the large argument limit of $\mathrm{K}_{0}$ can be used to simplify Eq. (28) to

$$
\begin{aligned}
& \left(\mathrm{T}-\mathrm{T}_{\infty}\right) /\left(\mathrm{T}_{\mathrm{M}}-\mathrm{T}_{\infty}\right)=\left(\pi \mathrm{R} /\left(\mathrm{P}\left(\mathrm{x}^{2}+\mathrm{y}^{2}\right)^{1 / 2}\right)\right)^{1 / 2} \exp \left(-\mathrm{P}\left(\left(\mathrm{x}^{2}+\mathrm{y}^{2}\right)^{1 / 2}\right.\right. \\
& +\mathrm{x}) /(2 \mathrm{R})) /(\varepsilon+\ln (2 / \mathrm{P}))
\end{aligned}
$$

Equation (29) indicates exponential decay of the recuced temperature along lines parallel and perpendicular to the weld seam except along the weld seam itself behind the pintool where the decay is algebraic like inverse square root of $\mathrm{x}$. This is a very slow decay and indicates the need for very long computational regions behind the pintool when numerical FSW-T simulations are performed.

All results given thus far represent the viewpoint of an observer moving with the pintool relative to a fixed workpiece (or, equivalently, describe a workpiece moving relative to a fixed pintool). The viewpoint of a fixed observer at a distance $\mathrm{X}$ to the right of point $i$ (see Fig. 1) on a fixed workpiece can be created by replacing $x$ in Eq. (28) by $\mathrm{X}-\mathrm{Vt}$ ( $\mathrm{t}$ being the time since process initiation) to get 


$$
\begin{aligned}
& \left(\mathrm{T}-\mathrm{T}_{\infty}\right) /\left(\mathrm{T}_{\mathrm{M}}-\mathrm{T}_{\infty}\right)=\mathrm{K}_{0}\left(\mathrm{P}\left((\mathrm{X}-\mathrm{Vt})^{2}+\mathrm{y}^{2}\right)^{1 / 2} /(2 \mathrm{R})\right) \exp (-\mathrm{P}(\mathrm{X} \\
& -\mathrm{Vt}) /(2 \mathrm{R})) /(\varepsilon+\ln (2 / \mathrm{P}))
\end{aligned}
$$

This describes a rapid increase in temperature as the pintool approaches followed by a slower decay as the pintool departs. This is in qualitative agreement with the experimental data presented in several of the previously cited references (see, for instance, figure 9 of [1], figure 13 of [7], figure 4 of [9], and figure 10 of [11]).

The simple form of Eq. (24) makes possible the definition of a sensitivity factor

$-\mathrm{S}=(\mathrm{d} \phi / \phi) /(\mathrm{d} \zeta / \xi)=(\xi / \phi)(\mathrm{d} \phi / \mathrm{d} \xi)=\xi /(1+\zeta)$

This can roughly be thought of as the ratio of the infinitesimal percentage change in $\phi$ to the infinitesimal percentage change in _ producing it. The sensitivity factor $\mathrm{S}$ is an increasing function of _ with a minimum value of zero and a maximum value of unity. A small percentage change in _ will, therefore, always produce a smaller percentage change in $\phi$. Thus, $\phi$ is not highly sensitive to small changes in . This allows some freedom in choosing the fitting parameter _. As a specific example, the parametric configurations to be discussed in the next section suggest that _ seldom exceeds $1 / 2$. The value corresponds to $S=1 / 3$, indicating that a small percentage change in _ in the vicinity of $1 / 2$ will produce a percentage change in $\phi$ one third as large.

\section{Numerical Examples}

Since the forms of the results presented in the previous section are so simple, it seem unnecessary to report extensive tabular or graphical representations of predictions. Nevertheless, a few numerical examples will be presented in this section with the purpose of investigating the issue of tuning. As mentioned earlier, no FSW-T model is fully 
determinate because the heating cannot be completely characterized without a knowledge of the mechanical behavior. For this reason, tuning is a feature of all FSW-T models (see, for instance, [1-4] and [6-8]). Several choices of tuning parameters have appeared in the literature. In the present simplified model there is no choice. The quantity _o,o must be selected. It is of interest, therefore, to determine the sensitivity of predictions to $\_0,0$ and to see if a value chosen on the basis of one set of experimental data can predict other sets of experimental data.

To deal with realistic configurations; it is necessary to select the correction factor $f$ to account for the actual pintool geometry shown in Fig. 2. There is no unique way to do this. Here it is accomplished by assuming that a constant transverse shear stress at the yield value is acting on the bottom and side surfaces of the nib and the bottom surface of the shoulder and finding the equivalent heat flux associated with the mechanical power due to the corresponding moment about the pintool centerline (similar to the methodology employed in [8]). Doing this shows that Eq. (4) must be replaced by

$\mathrm{q}_{\mathrm{n}}=\hat{\mathrm{o}}_{0} \mathrm{r} \Omega$

to take into account the variable moment arms on the nib and shoulder bottoms. In Eq. (32) $q_{n}$ is the heat flux normal to the pintool surface. According to this model, the net heating rate due to the mechanical dissipation is

$Q=\iint_{A} q_{n} d A$

where $\mathrm{A}$ is the combined nib bottom and side areas and the shoulder area. Carrying out this integration and defining an equivalent radial heat flux for the rotating plug model as $\mathrm{q}_{\mathrm{r}}(\mathrm{R}, \mathrm{è}, \mathrm{z})=\mathrm{Q} /(2 \partial \mathrm{RH})$ 
(the denominator of Eq. (34) being the rotating plug surface area) produces Eq. (4) with the $\hat{o}_{0}$ appearing therein increased by a multiplicative factor of

$\mathrm{f}=\mathrm{D} / \mathrm{H}+(\mathrm{R} / \mathrm{H})\left(\mathrm{R}_{\mathrm{S}} / \mathrm{R}\right)^{3} / 3$

For the full penetration configuration $(D=H)$ the corresponding result is

$f=1+(R / H)\left(\left(R_{S} / R\right)^{3}-1\right) / 3$

Four typical configurations based on the previously cited literature are characterized in columns 1-7 of Table 1. The values of $\mathrm{k}$ and a correspond to $6061 \mathrm{Al}$ which is the workpiece material in all five of these configurations. The value of $\hat{o}_{0,0}=$ 60.0 $\mathrm{MPa}=60.0 \mathrm{~N} / \mathrm{mm}^{2}$ was obtained by tuning to make the model predict temperatures in the FSW range. The need for such tuning in all FSW-T models was discussed earlier. Obviously, tuning can be done in many ways and no attempt to optimize the process was made herein.

Columns 8-11 of Table 1 show the values of pertinent intermediate quantities and column 12 shows the average reduced temperatures $(\phi)$ at the pintool surface. Column 13 contains the corresponding values of the predicted pintool surface average absolute temperature (T). The values reported in column 11 provide support for the estimate $<1 / 2$ used in the previous section.

Column 14 of Table 1 presents estimates of the observed maximum absolute temperatures $(T)$ in the experiments discussed in each of the references cited. Estimates are required both because it is possible to measure temperatures in only a limited number of locations in a FSW experiment (and practically impossible to measure the maximum temperature) and because not all data are reported in publications. Even allowing for 
some estimation error, it can be said that the agreement between predictions and observations is remarkably good, considering the simplicity of the model. It should be 'emphasized that a single value of _o,o has been used to make all five predictions. No attempt has been made to correct for the dependence of yield stress on strain rate or the fact that part of the pintool surface may be subjected to sliding friction.

\section{Conclusion}

In the forgoing, a simplified rotating plug model of friction stir welding heat transfer was used to develop a relatively simple closed form approximate solution predicting the workpiece temperature distribution produced by FSW. It was found that the temperature distribution in the immediate vicinity of the pintool could be characterized by a well known elementary conduction heat transfer solution. It was further observed that the average reduced temperature at the pintool surface depended on only one composite dimensionless parameter containing information about the translational and rotational weld speeds, the pintool geometry, and the workpiece material properties. The sensitivity to this parameter was found to be moderate. A single value of a tuning parameter was found to be able to produce predictions in good agreement with data from four different sets of FSW experiments involving $6061 \mathrm{Al}$ as the workpiece material. This suggests that once the model is tuned using one set of data for a particular workpiece material, it should be able to deal with other configurations pertinent to the same workpiece material. It is believed, therefore, that the present rotating plug FSW-T model should be useful for quick preliminary parametric studies of proposed FSW configurations. 


\section{Acknowledgement}

This research was supported by the National Aeronautics and Space Administration George C. Marshall Space Flight Center through Grant/Cooperative Number NCC8-223 for the National Center for Advanced Manufacturing - Louisiana Partnership. Any opinions, findings, and conclusions or recommendations expressed in this material are those of the authors and do not necessarily reflect the views of the National Aeronautics and Space Administration.

\section{Nomenclature}

$A=$ pintool area

$\mathrm{B}=$ Biot number

$D=$ pin depth

$\mathrm{f}=$ area correction factor

$\mathrm{H}=$ workpiece thickness

$\mathrm{h}=$ convection heat transfer coefficient

$\mathrm{K}_{\mathrm{o}}=$ zeroth order modified Bessel function of second kind

$\mathrm{L}=$ workpiece length

$\mathrm{k}=$ thermal conductivity

$\mathrm{P}=$ Peclet number

$\mathrm{p}=$ power

$\mathrm{Q}=$ heat source strength

$\mathrm{q}=$ heat flux

$\mathrm{R}=$ pin radius

$\mathrm{R}_{\mathrm{S}}=$ shoulder radius

$r=$ polar coordinate

$\mathrm{r}_{\mathrm{i}}=$ shell inner radius

$\mathrm{r}_{0}=$ shell outer radius

$\mathrm{S}=$ sensitivity factor

$\mathrm{T}=$ temperature

$\mathrm{T}_{\mathrm{M}}=$ melting temperature

$\mathrm{T}_{\mathrm{i}}=$ shell inner temperature 
$\mathrm{T}_{\mathrm{o}}=$ shell outer temperature

$\mathrm{T}_{\mathrm{A}}=$ anvil temperature

$T_{\infty}=$ environmental temperature

$t=$ time

$V=$ pintool velocity

$\mathrm{W}=$ workpiece width

$X=$ cartesian coordinate

$\mathrm{x}=$ cartesian coordinate

$y=$ cartesian coordinate

$\mathrm{z}=$ cartesian or polar coordinate

-thermal diffusivity

-mechanical dissipation number

= composite dimensionless parameter

_-polar coordinate

${ }_{0}=$-yield stress

$-0,0=$ fitting parameter

$\phi=$ average reduced pin temperature

_-reduced temperature function

${ }_{-}$= shell reduced temperature function

_-pintool angular velocity

subscripts

$\mathrm{H}=$ workpiece top

$\mathrm{n}=$ normal

$\mathrm{o}=$ workpiece bottom

r=radial

$\mathrm{z}=\mathrm{axial}$

-transverse 


\section{References}

[1] Chao, Y. J., and Qi, X. H., Thermal and Thermo-Mechanical Modeling of Friction Stir Welding of Aluminum Alloy 6061-T6, Journal of Material Processing and Manufacturing Science, Vol.7, pp. 215-233, 1994.

[2] Ulysse, P., 2002, Three-Dimensional Modeling of the Friction Stir-Welding Process, International Journal of Machine Tools and Manufacture, Vol. 42, pp 1549-1557, 2002.

[3] Hyoe, T., Colegrove, P.A., and Shercliff, H.R., Thermal and Microstructure Modeling in Thick Plate Aluminum Alloy 7075 Friction Stir Welds, Friction Stir Welding and Processing, Vol. 2, pp. 33-42, 2003.

[4] Chao, Y.J., Qi, X.H., and Tang, W., Heat Transfer in Friction Stir Welding Experimental and Numerical Studies, ASME Journal of Manufacturing Science and Engineering, Vol. 125, pp. 138-145, 2003.

[5] Colegrove, P.A., and Shercliff, H.R., 2-Dimensional CFD Modeling of Flow Round Profiled FSW Tooling, Friction Stir Welding and Processing, Vol. 2, pp. 13-22, 2003.

[6] Song, M., and Kovacevic, R., Numerical and Experimental Study of the Heat Transfer Process in Friction Stir Welding, Journal of Engineering Manufacture, Vol: 217 , pp. 73-85, 2003.

[7] Song, M. and Kovacevic, R., Thermal Modeling of Friction Stir Welding in a Moving Coordinate System and its Validation, International Journal of Machine Tools and Manufacture, Vol. 43, pp. 605-615, 2003.

[8] Schmidt, H, Hattel, J., and Wert, J., An Analytical for the Heat Generation in Friction Stir Welding, Modelling and Simulation in Materials. Science and Engineering, Vol. 12, pp.143-157, 2004.

[9] Chen, C. and Kovacevic, R., Thermomechanical Modelling and Force Analysis of Friction Stir Welding by the Finite Element Method, Journal of Mechanical Engineering Science, Vol. 218, pp. 509-519, 2004.

[10] Soundararajan, V., Zekovic, S., and Kovacevic, R., Thermo-Mechanical Model with Adaptive Boundary Conditions for Friction Stir Welding of Al 6061, International Journal of Machine Tools and Manufacture, Vol. 45, pp. 1577-1587, 2005.

[11] Schmidt, H. and Hattel, J., A Local Model for the Thermomechanical Conditions in Friction Stir Welding, Modelling and Simulation in Materials Science and Engineering, Vol: 13, pp. 77-93, 2005. 
[12] Colegrove, P.A., and Shercliff, H.R., 3-Dimensional CFD Modelling of Flow Round a Threaded Friction Stir Welding Tool Profile, Journal of Materials Processing Technology, Vol. 169, pp. 320-327, 2005.

[13] Cho, J.H., Boyce, D.E., and Dawson, P.R., Modeling Strain Hardening and Texture Evolution in Friction Stir Welding of Stainless Steel, Materials Science and Engineering, Vol. 398A, pp. 146-163, 2005.

[14] Zhang, H.W., Zhang, Z., and Chen, J.T., The Finite Element Simulation of the Friction Stir Welding Process, Materials Science and Engineering, Vol. 403A, pp. 340-348, 2005.

[15] Carslaw, H.S., and Jaeger, J.C., Conduction of Heat in Solids, Oxford University Press, London, 1947.

[16] Trivedi, R and Srinivasan, S.R., Temperature Distribution Around a Moving Cylindrical Source, ASME Journal of Heat Transfer, Vol. 96, pp. 427-428, 1974.

[17] Incropera, F.P. and Dewitt, D.P., Fundamentals of Heat and Mass Transfer, Fourth Edition, Wiley, New York, 1996. 
Figure captions

Fig. 1 Schematic of typical friction stir welding configuration

Fig. 2 Schematic of a typical pintool (partial penetration configuration) 


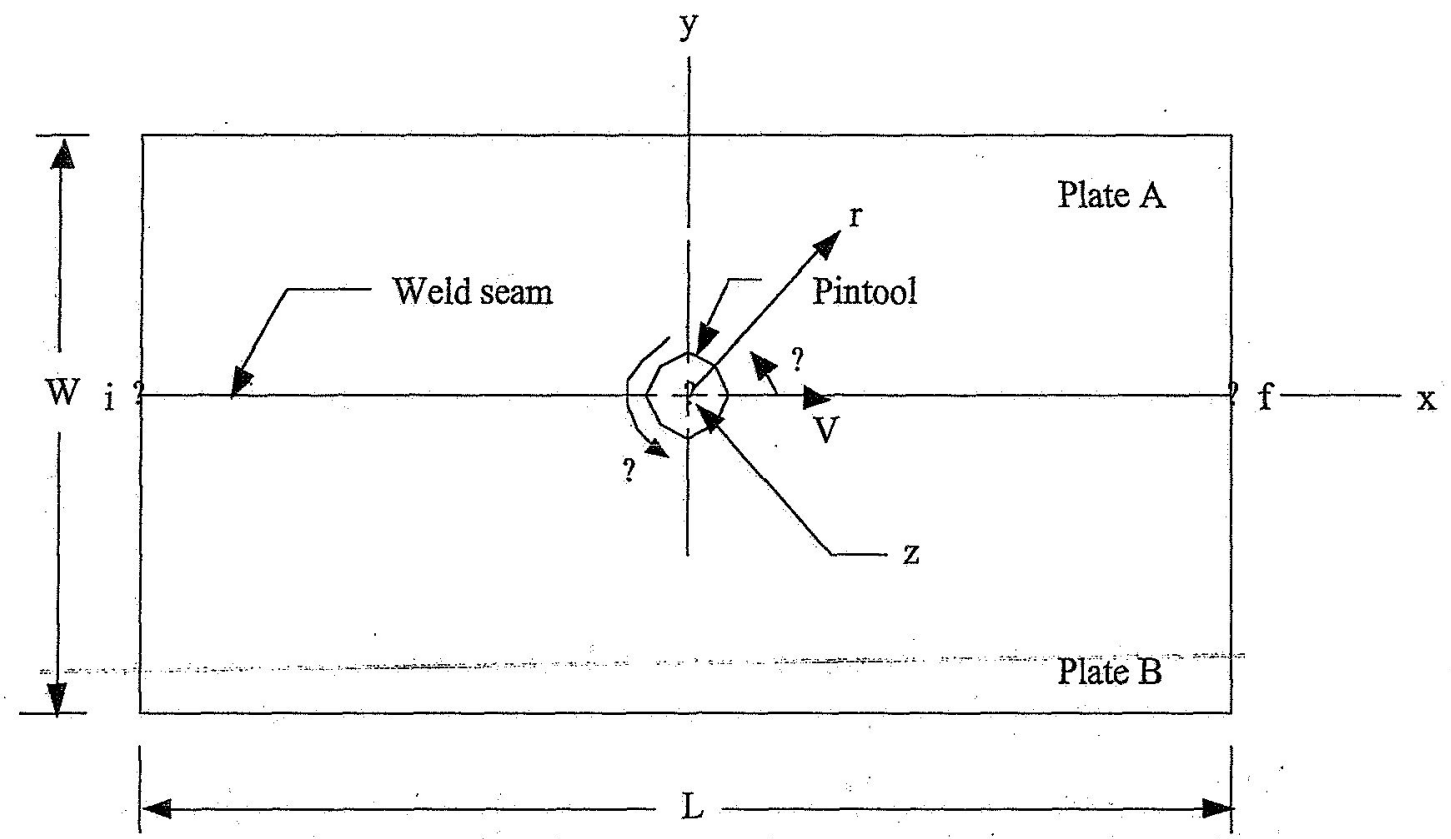

Fig. 1 Schematic of typical friction stir welding configuration 


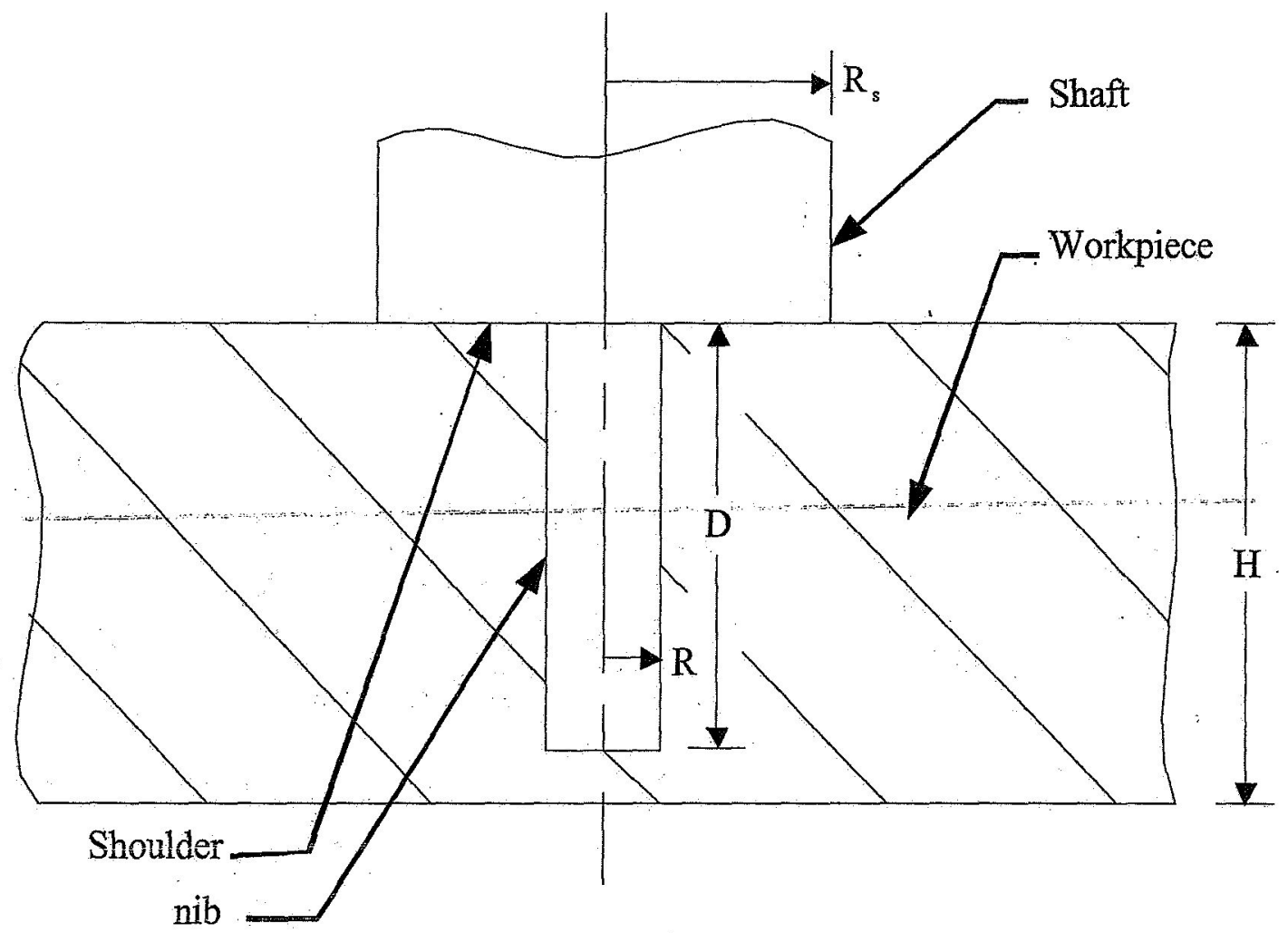

Fig. 2 Schematic of a typical pintool (partial penetration configuration) 


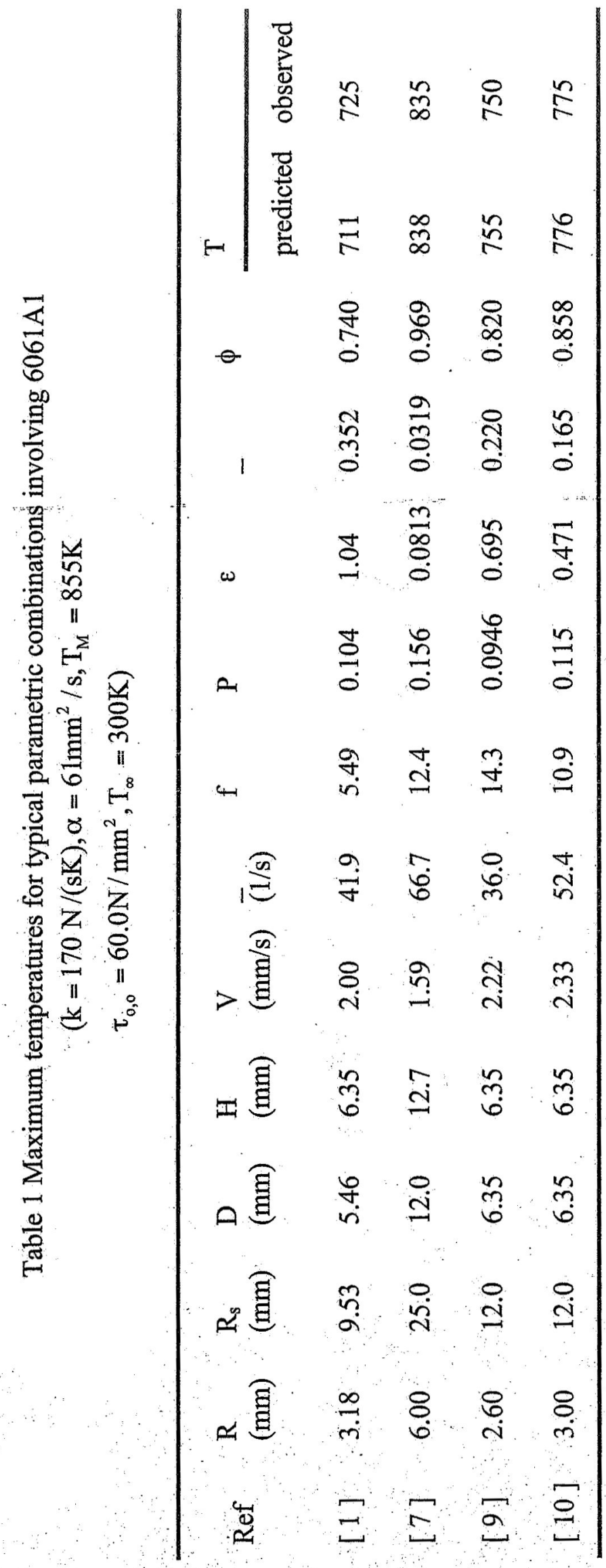

